\title{
L'évaluation des trames vertes urbaines : apports et limites de la socio-économie des transports
}

Evaluation of Urban Green Spaces: Contributions and Limitations of the Socioeconomics of Transport

\section{Jacques Stambouli}

\section{(2) OpenEdition}

\section{Journals}

Édition électronique

URL : http://journals.openedition.org/developpementdurable/9417

DOI : 10.4000/developpementdurable.9417

ISSN : 1772-9971

Éditeur

Association DD\&T

\section{Référence électronique}

Jacques Stambouli, «L'évaluation des trames vertes urbaines : apports et limites de la socio-

économie des transports », Développement durable et territoires [En ligne], Vol. 3, n 3 | Décembre 2012, mis en ligne le 22 novembre 2012, consulté le 03 mai 2019. URL : http://journals.openedition.org/ developpementdurable/9417; DOI : 10.4000/developpementdurable.9417

Ce document a été généré automatiquement le 3 mai 2019.

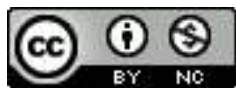

Développement Durable et Territoires est mis à disposition selon les termes de la licence Creative Commons Attribution - Pas d'Utilisation Commerciale 4.0 International. 


\title{
L'évaluation des trames vertes urbaines : apports et limites de la socio-économie des transports
}

\author{
Evaluation of Urban Green Spaces: Contributions and Limitations of the \\ Socioeconomics of Transport
}

Jacques Stambouli

1 Une trame verte est constituée, selon la définition du programme de recherche ANRTrame Verte Urbaine, «de grands ensembles naturels et de corridors les reliant ou servant d'espaces tampons ». Pour les trames vertes urbaines, ces corridors et ces ensembles naturels sont situés dans l'espace urbain, car ce dernier a tendance à fragmenter les ensembles naturels. Les trames vertes urbaines peuvent ensuite se prolonger dans les espaces ruraux et naturels qui entourent les espaces urbains.

2 L'objectif premier d'une trame verte est de préserver ou d'augmenter la qualité et la quantité des habitats naturels et des connexions entre ces habitats. Les habitats naturels jouent le rôle de réservoirs ou de stocks de gènes, d'espèces, de communautés d'êtres vivants, c'est-à-dire de réservoirs de biodiversité. L'augmentation de ces habitats a pour effet de préserver, voire d'augmenter la biodiversité sur un territoire donné. Cette préservation/augmentation de la biodiversité, liée à la trame verte, servira en même temps, à l'initiative des collectivités territoriales, à améliorer le cadre de vie pour les habitants concernés, ce qui se traduira par des flux de services fournis aux habitants.

Une trame verte urbaine correspond donc à un projet d'aménager des corridors reliant des habitats naturels, sous la responsabilité des collectivités territoriales. Ces corridors sont des infrastructures, éléments de base fixes, permettant des flux d'espèces animales et végétales et des flux de services pour les sociétés humaines liés à ces espèces animales et végétales. Cette terminologie (corridors, infrastructures aménagées, flux, amélioration du cadre de vie, rôle des collectivités territoriales) rappelle celle des infrastructures de transport, éléments fixes d'un réseau permettant la circulation des personnes et des biens à l'intérieur de territoires donnés et ayant des effets potentiels d'aménagement de ces 
territoires par le jeu des acteurs de ces territoires. L'évaluation socio-économique par les collectivités territoriales, maitres d'ouvrage, des projets d'infrastructures de transport, du fait de l'importance des dépenses publiques engagées, a fait l'objet, depuis des décennies en France et dans de nombreux pays, de méthodes normalisées d'évaluation socio-économique (Commissariat Général du Plan, 2001; Conseil Général des Ponts et Chaussées, 2005 ; Ministère des Transports, 2005 ; Schiller et al., 2010 ).

L'aménagement des corridors des trames vertes urbaines, et éventuellement le réaménagement des espaces naturels à relier, en fonction de l'existence de ces corridors, demanderont aussi, comme les infrastructures de transport, des investissements publics, qui devront être justifiés, en particulier de façon monétaire. Il est donc utile de prendre en compte les différentes méthodes d'évaluation socio-économiques des infrastructures de transport, pour déterminer leurs apports pour une évaluation des trames vertes urbaines, ce que nous ferons dans un premier temps. Nous aborderons ensuite, dans un deuxième temps, les limites de ces évaluations pour les trames vertes urbaines, dans la mesure où les rôles des deux types d'infrastructures étudiées et comparées sont en bonne partie différents et où les évaluations socio-économiques ne constituent qu'un type particulier d'évaluation dans une perspective de développement durable.

\section{Les apports de l'évaluation socio-économique des infrastructures de transport pour les trames vertes urbaines.}

\subsection{L'analyse coûts-avantages et ses limites}

5 En France et dans de nombreux pays, l'évaluation des projets d'infrastructures de transport a lieu dans le cadre d'une analyse coûts-avantages. Les coûts du projet d'aménagement sont comparés aux avantages du projet, en valorisant monétairement coûts et avantages. Si les avantages monétarisés l'emportent sur les coûts, ce qui se traduit par l'existence d'un bénéfice social, le projet peut être adopté. Le processus d'évaluation se fait en deux étapes. Dans une première étape, un débat public « d'amont » est couplé à une évaluation socio-économique approchée. Cette étape sert «à préciser les finalités du projet, (...) à faire l'inventaire des variantes envisageables, à distinguer les différents niveaux des collectivités locales impliquées, à identifier les groupes réellement concernés ainsi que leurs enjeux légitimes. (...) Dans la seconde étape, l'évaluation socio-économique devrait progressivement s'affiner pour aboutir (...) à un calcul de bilan socio-économique » (Ministère des Transports, $2005: 12$ ).

6 Cette méthode apparaît intéressante, dans un premier temps, pour les trames vertes urbaines. Comme projet d'aménagement, la trame verte urbaine comporte nécessairement un coût monétaire, supporté notamment par la ou les collectivités territoriales, sous forme de dépenses d'investissements et de fonctionnement. Il est logique de comparer ce coût de la trame verte urbaine aux avantages qu'elle devrait apporter. Il faut donc essayer de monétariser ces avantages, même si ces derniers ne sont pas monétaires d'emblée, afin de disposer d'une base commune d'évaluation par rapport aux coûts. Les avantages de la trame verte, comme dans le cas des infrastructures de transport, seront des avantages nets, ce qui permettra d'intégrer l'augmentation possible d'une biodiversité nuisible pour les êtres humains, valorisée négativement : par exemple 
des risques d'accidents liés à une biodiversité "sauvage " pour les humains ou à des piqûres d'insectes, qui peuvent être évalués, selon le coût des dommages par exemple.

7 Les infrastructures de transport ont des effets souvent négatifs sur l'environnement naturel, tel qu'il est perçu par les sociétés humaines : bruit, pollution atmosphérique locale ou régionale, effet de serre. Ces impacts sont valorisés selon différentes méthodes : coûts des dommages ou des dépréciations créées pour les riverains; coûts d'évitement des dommages ; préférences révélées par les marchés de substitution, les prix hédoniques ou préférences déclarées par les évaluations contingentes (Quinet, 1998 ; Conseil National des Transports, 1999 : ; Stambouli, 2000 ). Le Ministère des Transports propose des valeurs "tutélaires", en combinant différentes méthodes, pour les effets indirects ou non marchands: gains de temps, accidents, bruit, pollution locale et régionale de l'air, émissions de $\mathrm{CO} 2$ afin de comparer les projets d'infrastructures de transport entre eux.

8 Les trames vertes urbaines ont aussi des effets sur l'environnement naturel perçu par les sociétés humaines, concernant le bruit, la qualité locale ou régionale de l'air ou les émissions de $\mathrm{CO}_{2}$. Mais ces effets sont plutôt positifs : baisse du bruit, filtrage de l'air, absorption $\mathrm{du} \mathrm{CO}_{2}$ par les plantes par exemple. Ces effets pourraient être valorisés selon des méthodes comparables à celles des infrastructures de transport, en calculant par exemple une diminution des dommages par rapport à une situation de référence. Des résultats différents, selon les méthodes utilisées, pourraient donner lieu à des fourchettes d'évaluation des effets des trames vertes sur l'environnement naturel.

9 L'augmentation possible des loyers, calculée par exemple par la méthode des prix hédoniques, liée à l'amélioration du cadre de vie du fait de la trame verte urbaine ou d'une infrastructure de transport, est un effet négatif pour les locataires, mais un effet positif pour les propriétaires des logements concernés. Mais une politique de logement social, de la part d'une collectivité locale, peut limiter les augmentations de loyers liées à l'amélioration du cadre de vie. Cet exemple illustre la pluralité des acteurs concernés, leurs intérêts parfois divergents d'un point de vue socio-économique, et la nécessité d'études sociales précises pour déterminer les effets réels d'une trame verte dans un lieu donné.

10 On touche là à certaines limites de l'analyse coûts-avantages, que ce soit pour les infrastructures de transport ou pour les trames vertes urbaines. Comme l'explique Pascal Bérion (2007: 651-676), " une méthode coûts-avantages (...) sous-tend une relation de type cause/conséquence (directe ou conditionnelle) entre réalisation de l'infrastructure et comportement économique. (...) Elle tend à offrir une vision abstraite de l'infrastructure, exprimée par une rentabilité (...) sans lien avec les mutations du territoire proprement dit ». Il ne s'agit pas de rejeter la notion d'effet potentiel de l'infrastructure et les valorisations qui en découlent. Mais, dans le cas des infrastructures de transport, comme dans le cas des trames vertes urbaines, comme l'explique Pascal Bérion, « la recherche doit se concentrer sur les usages et les représentations de l'infrastructure par les agents économiques " afin de déterminer ce qu'ils en font réellement, ce qu'ils veulent en faire et en tenant compte des interactions possibles entre les acteurs induisant des mutations possibles des territoires. Pour la trame verte urbaine, les usages et les représentations peuvent être très variés, du fait de la diversité des acteurs et de leurs finalités, bien plus que pour les infrastructures de transport qui ont une finalité essentiellement liée à la mobilité des personnes et des biens. Il est donc encore plus nécessaire pour les trames vertes urbaines que pour les infrastructures de transport de dépasser le cadre de l'analyse coûts-avantages pour prendre en compte la diversité des acteurs, leurs finalités et leurs interactions. 


\subsection{L'intérêt d'une analyse multicritères spatialisée}

11 L'analyse multicritères est utilisée quand il est reconnu qu'il y a des coûts et des bénéfices non monétarisables pour un projet donné : par exemple des impacts culturels, liés aux paysages, à la perception de l'environnement par différents acteurs (usagers, agriculteurs, aménageurs, associatifs dans le cas des trames vertes) ou des impacts sociaux contradictoires liés à des conflits d'usages. C'est le cas pour les infrastructures de transport, comme pour les trames vertes urbaines. Une analyse multicritères inclut, pour les infrastructures de transports, différentes catégories d'impacts qui ne sont pas nécessairement monétarisés: environnementaux, sociaux ou de développement économique de long terme (Schiller et al., 2010).

12 Au Royaume Uni, depuis la fin des années 1990, les infrastructures de transport sont évaluées selon une analyse multicritères non agrégée, en milieu interurbain et urbain, incluant des éléments monétaires et non monétaires (Conseil Général des Ponts et Chaussées, 2005).

13 En France, le rapport environnemental sur le Schéma National des Infrastructures de Transport, établi pour le Ministère de l'Écologie est aussi basé sur une grille d'analyse multicritères (Commissariat Général du Développement Durable, 2011). Les axes, les risques et les coûts du Schéma National des Infrastructures de Transport sont évalués par des indicateurs souvent spatialisés. Par exemple, le risque environnemental et biologique lié aux infrastructures de transport projetées est décliné sous forme de sous-objectifs dont celui de préserver les espaces naturels répertoriés, avec comme indicateur le linéaire d'infrastructures au droit de ces espaces naturels. Cet indicateur permet ensuite d'agir spatialement, en évitant, réduisant ou compensant le risque lié à l'infrastructure de transport. La définition des trames verte et bleue apparait alors comme l'un des moyens de réduire les impacts environnementaux négatifs des infrastructures de transport dans le cadre d'une analyse multicritères spatialisée, en préservant ou en augmentant la biodiversité d'espaces naturels protégés qui seraient fragmentés par les projets d'infrastructures de transport.

14 Du fait d'impacts environnementaux, sociaux, culturels, de finalités contradictoires, souvent non monétarisables, et en cohérence avec leur rôle vis-à-vis des grandes infrastructures de transport qui ont un impact le plus souvent négatif sur la biodiversité suivant l'analyse multicritères du Ministère de l'Écologie, il est donc intéressant d'évaluer les trames vertes urbaines dans le cadre d'une analyse multicritères spatialisée.

\subsection{La comparaison de deux situations : une situation de référence sans le projet et une situation avec le projet}

15 L'évaluation des projets d'infrastructures de transport compare la situation optimisée la plus probable en l'absence de projet, pour un horizon temporel défini, et la situation avec le projet suivant le même horizon temporel. La méthode apparaît adéquate pour les trames vertes urbaines. Il est possible de comparer une situation de référence avec des habitats naturels non reliés; et une situation avec les mêmes habitats naturels complétés par des corridors écologiques. La situation de référence sans le projet doit intégrer l'évolution de l'espace de référence sur plusieurs dizaines d'années avec les risques de pertes de biodiversité. Elle doit être comparée à la situation avec le projet. Le coût du 
projet de trame verte peut se justifier simplement par la valorisation de l'absence des pertes prévisibles de biodiversité, à condition de valoriser cette biodiversité, ce qui sera abordé plus loin, dans une partie sur les services écosystémiques liés à la biodiversité.

\subsection{L'utilisation d'un taux d'actualisation, marquant une préférence pour le présent}

16 Comme les avantages d'une infrastructure de transport et ceux d'une trame verte urbaine sont de longue durée, il est logique d'introduire un taux d'actualisation pour les avantages et les coûts monétarisés. Ce taux d'actualisation traduit une préférence de la société pour les avantages présents par rapport aux avantages à venir, en partant du moment où l'investissement pour l'aménagement est dépensé. Les avantages divers d'une trame verte urbaine sont effectivement étalés dans le temps, que ce soit l'amélioration de la biodiversité ou du cadre de vie. Il serait même logique, afin de comparer des projets de trame verte, de prendre le même taux d'actualisation pour tous les projets. Cependant, pour prendre en compte des générations futures, les taux d'actualisation doivent être faibles, pour ne pas réduire à néant un éventuel avantage dans vingt-cinq ou cinquante ans.

\subsection{L'utilisation de la valeur du temps, dans le cas des modes " actifs » utilisant les trames vertes}

Les trames vertes urbaines peuvent être utilisées comme infrastructures de transport des personnes et des marchandises, à condition que ces transports ne constituent pas des obstacles aux flux de la faune et de la flore. C'est pourquoi, pour les déplacements des personnes, on ne pourra considérer comme trames vertes urbaines que des infrastructures qui peuvent être utilisées pour la marche ou la bicyclette; et pour le transport des marchandises et des personnes, on ne prendra en compte, comme trames vertes, que des espaces fluviaux ou maritimes.

Pour tous ces modes de transport (marche, bicyclette, transport fluvial ou maritime), on peut estimer que les atteintes à la flore et à la faune peuvent être minimisées dans l'état des techniques actuelles. Pour les transports de personnes, l'automobile individuelle, les deux- roues motorisés, les transports collectifs routiers ou ferroviaires sont actuellement générateurs de pollutions diverses, de bruit, de déplacements très rapides qui perturbent profondément la faune et la flore. Pour les transports de marchandises, ces nuisances sont associées aux transports routiers, ferroviaires ou aériens. Les conduites de pétrole ou de gaz nous semblent aussi peu compatibles avec des trames vertes en ville, du fait des risques associés de pollutions.

Comme infrastructures de transport, les trames vertes urbaines peuvent apporter un gain de temps aux usagers pour leurs déplacements, par exemple un chemin plus court pour la marche ou la bicyclette, limitant les effets de coupure urbaine. Ce gain de temps peut être valorisé selon la valeur du temps donnée dans l'évaluation des infrastructures de transport : en moyenne 7,6 euros (2000) de l'heure en milieu urbain, en France, avec des valorisations supérieures pour des déplacements professionnels ou inférieures pour des déplacements de loisirs (Ministère des Transports, 2005). Ces valorisations sont établies à partir d'enquêtes socio-économiques sur des déplacements en voiture particulière utilisant des infrastructures de transport à péage, comparées à des déplacements du 
même type sans péage. Elles sont normalisées en France : leur normalisation peut aussi servir pour des déplacements utilisant d'autres modes de transport, en supposant que les personnes donnent une valeur identique à leur temps, quel que soit le mode utilisé.

Certaines trames vertes ont pu être justifiées par l'aménagement de pistes cyclables (Montpellier, Strasbourg...) ou de cheminements piétons plus agréables ou plus pratiques. Les trames vertes urbaines, quand elles s'accompagnent de liaisons douces, piétonnes ou cyclables, de sentiers de randonnée, permettent alors plutôt un gain d'accessibilité à des espaces naturels remarquables, agréables en eux-mêmes. Ces espaces n'auraient pas été normalement accessibles, ou du moins dans des conditions très difficiles, caractérisées par des temps d'accès très longs. Dès lors, la valeur du temps gagné doit être appréciée comme un gain de temps pour accéder à des espaces agréables à partir d'un point donné : par exemple un gain d'accessibilité à divers lieux remarquables de la trame verte à partir du centre d'un quartier dans une région urbaine donnée. Ce gain d'accessibilité à un lieu naturel remarquable ne sera pas ainsi confondu avec un gain de temps dans un budgettemps fixé pour les déplacements de la vie quotidienne; car ce type de gain de temps favorise l'étalement urbain, qui détruit des espaces naturels nécessaires à la trame verte.

\subsection{La valorisation de modes de transport « actifs » pour les personnes par rapport au transport automobile.}

21 Les trames vertes urbaines sont compatibles avec des modes "actifs ", non motorisés, comme la marche ou la bicyclette. Elles remettent en cause, dans la situation actuelle de nos villes, les modes motorisés, en particulier l'automobile individuelle dans les espaces qu'elle occupe. Une comparaison entre deux situations, l'une avec la trame verte, l'autre sans, peut montrer une réduction de l'espace utilisé par l'automobile individuelle, et donc une réduction des nuisances liées à ce mode de transport: accidents de la circulation, bruit, pollution locale, effet de serre dont nous avons mentionné la valorisation monétaire mais aussi effets de coupure des espaces, consommation des espaces publics, dégradation des paysages, pollution des eaux, des sols... Ces derniers effets sont rarement évalués dans les analyses coûts-avantages et pourront faire l'objet d'évaluations non monétaires avec des indicateurs dans le cadre d'une analyse multicritères spatialisée.

De plus, selon Frédéric Héran (2011), les nuisances liées au transport automobile font système: elles interagissent entre elles, pour renforcer la nécessité du transport automobile et contribuer à une désaffection pour les modes actifs, à une diminution des relations de voisinage, à une dégradation de la santé humaine et du cadre de vie. Une remise en cause de l'espace de l'automobile en ville par les trames vertes urbaines peut alors aussi faire système en sens inverse : augmentation de la fréquentation des modes actifs, des relations de voisinage, amélioration de la santé humaine et du cadre de vie. Un élément nouveau, non encore évalué précédemment, apparaît alors: l'augmentation possible des relations de voisinage liées à la trame verte, que l'on peut mesurer par la fréquentation de cet espace, si c'est un espace public. L'évaluation monétaire de cette fréquentation pourrait se faire par la méthode des marchés de substitution, en calculant les coûts de voyages pour atteindre la trame verte urbaine, comme dans le cas de la valorisation d'un espace naturel remarquable. Cependant, comme il s'agit de relations de voisinage, les coûts des voyages seront faibles. Nous atteignons là encore les limites de la valorisation monétaire pour la trame verte urbaine: il vaudra mieux donner comme 
indicateur une fréquentation générale non monétarisée et s'intéresser aux différents acteurs fréquentant la trame verte et à leurs usages.

\subsection{La prise en compte de différents types d'acteurs à différentes échelles}

Les effets des infrastructures de transport sont évalués du point de vue de la collectivité territoriale pour trois types d'acteurs : les utilisateurs de ces infrastructures, des autres modes de transport et les tiers concernés; les entreprises de transport et les gestionnaires d'infrastructures; l'État et les collectivités territoriales. Dans le cas des trames vertes urbaines, cette distinction peut être reprise en partie. Il y a effectivement des utilisateurs de la trame verte, si celle-ci constitue un espace vert public (par exemple familles avec enfants, promeneurs, sportifs), un lieu de production de biomasse (pour le bois-énergie ou les composts des administrations des parcs et jardins), un lieu d'utilisation de services divers (culturels, récréatifs, éducatifs). Les riverains de la trame verte sont concernés par la baisse éventuelle du bruit, une meilleure qualité de l'air, qui peuvent avoir un effet positif sur la valorisation de leur patrimoine immobilier et sur leur santé. Des tiers peuvent être concernés, du fait des espaces impactés à différentes échelles : agriculteurs ou jardiniers bénéficiant de la pollinisation des plantes, chasseurs ou pêcheurs bénéficiant de l'augmentation de la biodiversité etc. L'État et surtout les collectivités territoriales sont aussi concernés en matière de dépenses ou de recettes (variations d'impôts ou de taxes). Les entreprises de transport ne sont pas concernées pour l'essentiel, sauf si la trame verte sert aussi d'infrastructure de transport, par exemple pour les marchandises (transport fluvial ou maritime) ou pour les personnes (cas de la bicyclette).

En conclusion, différents effets environnementaux potentiels de la trame verte urbaine (diminution du bruit, de la pollution locale de l'air, des émissions de $\mathrm{CO}_{2}$ ), des effets sociaux éventuels (risques d'accidents) et des améliorations possibles de la mobilité «active» (gains de temps) peuvent être évalués monétairement par les méthodes traditionnelles de la socio-économie des transports, à condition de bien identifier les acteurs concernés et les usages réels ou potentiels de la trame verte urbaine. Cependant parce qu'elles induisent des impacts culturels (par exemple sur les paysages), environnementaux (par exemple de préservation d'espaces naturels remarquables) et sociaux (conflits d'usages) souvent non monétarisables, avec une interaction systémique d'acteurs dans un cadre spatialisé, les trames vertes urbaines nécessitent une évaluation multicritères spatialisée, combinant des éléments monétaires et non monétaires, conformément à ce qui est pratiqué dans certains cas pour les infrastructures de transport.

\section{Limites de la socio-économie des transports. Les services liés à la biodiversité, les services liés à l'aménagement et les différentes échelles d'aménagement}

Les utilisateurs de la trame verte urbaine ne l'utilisent pas essentiellement comme une infrastructure de transport, espace artificiel aménagé pour les déplacements. La trame 
verte urbaine est un espace naturel aménagé dont l'objectif premier est la préservation ou l'augmentation de la biodiversité. Nous allons étudier les services spécifiques liés à cet objectif premier pour différents acteurs, en continuant notre analyse comparative par rapport à une infrastructure de transport. Nous soulignerons alors les limites des évaluations de la socio-économie des transports pour les trames vertes urbaines et définirons quelques pistes pour leur évaluation socio-économique.

\subsection{Une démarche différente pour l'évaluation : partir des fonctions de base des écosystèmes avec des indicateurs écologiques}

Les corridors d'une infrastructure de transport ont pour objectif de favoriser des déplacements le long de cette infrastructure. L'évaluation socio-économique part donc des services de transport prévus sur cette infrastructure en y ajoutant des effets potentiels en fonction du jeu des acteurs concernés. Les corridors des trames vertes urbaines ont pour but de préserver ou d'augmenter la biodiversité. L'évaluation socioéconomique doit donc partir de cet objectif qui apportera des services éventuels pour les personnes concernées. Or si le déplacement des personnes et des biens constitue effectivement un service économique, pouvant avoir une valeur monétaire sur un marché, la biodiversité, comme situation des espèces de la biosphère, ne constitue pas en soi un bien économique, au sens de bien utile aux sociétés humaines.

7 Ce sont les usages de la biodiversité par les sociétés humaines qui pourront constituer des biens économiques, qu'ils soient matériels (par exemple l'usage du bois ou du miel) ou immatériels (par exemple des services éducatifs ou culturels) ou une combinaison des deux (par exemple du miel et des services éducatifs concernant les ruches et les abeilles). Dans la suite de l'article, nous appellerons services écosystémiques les usages des écosystèmes par les sociétés humaines, dont les usages de la biodiversité.

Comme le constate le Centre d'Analyse Stratégique (CAS, 2009: 169), «la biodiversité apparaît comme une propriété émergente d'autres éléments considérés comme des ressources: les écosystèmes, auprès desquels les agents économiques se fournissent en de multiples services. Ce sont donc plutôt les services écosystémiques qui peuvent être considérés comme des biens économiques. (...) Lorsqu'il est question de "valeur de la biodiversité", c'est donc principalement de la mesure de l'impact de la variation du paramètre biodiversité sur la valeur sociale des services écosystémiques qu'il s'agit ».

Le CAS reprend les travaux du Millenium Ecosystem Assessment (MEA) de 2005, qui a distingué des fonctions de base des écosystèmes et les services écosystémiques, apportés par ces fonctions de base. Une équipe d'écologues a étudié les fonctions de base des écosystèmes en France (Commissariat Général du Développement durable, mai 2010). Cette équipe a distingué 9 classes de milieux de vie ( 7 classes de milieux naturels, plus les espaces agricoles cultivés et les habitats artificialisés) et 13 fonctions de base liées à l'ensemble de ces milieux de vie. Les fonctions de base sont des processus bio-géochimiques qui se déroulent dans ces milieux de vie en dehors de l'intervention humaine, même s'ils peuvent être influencés par cette intervention. Ils vont des échanges gazeux végétation atmosphère, en passant par la décomposition de la matière organique jusqu'aux relations entre des habitats spécifiques pour la reproduction des espèces. Il est possible de proposer des indicateurs écologiques montrant l'état des fonctions écologiques de base dans ces milieux de vie, notamment à partir d'espèces servant de témoins. Des valeurs seuils peuvent être associées à ces indicateurs : « les valeurs seuils sont 
les limites en-deçà desquelles le bon fonctionnement de l'écosystème considéré n'est plus assuré. Les fonctions y sont dégradées et les services rendus de plus en plus faibles. La résilience de l'écosystème est donc maintenue tant que ces valeurs seuils ne sont pas atteintes" (Commissariat Général du Développement durable, mai 2010 : 22). Avant même d'évaluer des services écosystémiques liés à la biodiversité, il est donc nécessaire d'utiliser des indicateurs sur l'état des fonctions écologiques de base dans les milieux de vie qui fourniront ces services. L'objectif de la trame verte urbaine sera, dans des milieux de vie donnés, de préserver ou d'améliorer un certain nombre de ces fonctions de base, évaluées par des indicateurs écologiques, pour fournir des services écosystémiques liés aux milieux de vie à préserver.

\subsection{La diversité des services écosystémiques et l'importance d'une analyse multicritères pluraliste}

Les services des infrastructures de transport sont d'abord des services de déplacement. Les services potentiels d'une trame verte sont beaucoup plus diversifiés. Les services écosystémiques liés aux milieux de vie reliés par la trame verte sont, selon le MEA, à partir des fonctions de base, de trois types: des services de régulation, des services d'approvisionnement et des services culturels. Les services de régulation concernent la capacité de moduler dans un sens favorable aux sociétés humaines des phénomènes naturels liés à la biodiversité qui se déroulent sans une intervention humaine : régulation de la qualité de l'air, de l'eau, du climat, des cycles de l'eau, des déchets, pollinisation, régulation des maladies et conservation des espèces par exemple. Les écologues sollicités par le Commissariat Général au Développement durable ont recensé 18 services écosystémiques de régulation pour les milieux de vie existant en France. Dans le cas de la trame verte, pour ce genre de services, on peut évaluer le coût des dommages si la trame verte n'est pas aménagée et le coût d'évitement des dommages par l'aménagement de la trame verte. L'aménagement de la trame verte permet de restaurer ou d'améliorer certaines fonctions fondamentales d'un milieu de vie pour lui permettre d'atteindre ou de dépasser les valeurs seuils. On peut mesurer aussi, par la méthode des préférences révélées (marchés de substitution ou prix hédoniques), la valeur marchande attribuée aux améliorations des services de régulation, par exemple pour la régulation de la qualité de l'air ou des risques d'inondations. Mais cette méthode ne relie pas nécessairement la valeur marchande attribuée à ces services aux seuils nécessaires pour le maintien du bon fonctionnement des milieux de vie à préserver. On peut aussi s'appuyer sur les préférences déclarées, par exemple sur un consentement à payer par les personnes concernées pour restaurer une meilleure qualité de l'air ou une meilleure prévention contre les inondations. Cependant, comme le note un rapport du Commissariat Général au Développement durable (CGDD, $2010: 31)$ : « les méthodes qui s'appuient sur les préférences déclarées dépendent de l'information que les agents détiennent sur la biodiversité. Or pour certains types de services rendus par la biodiversité, à l'exception notable des services paysagers et récréatifs par exemple, cette connaissance est souvent lacunaire, ce qui conduit à une plus grande imprécision dans les résultats que pour d'autres objets d'études». Pour les services de régulation, les évaluations monétaires se combineront donc avec des indicateurs écologiques de bon fonctionnement des milieux de vie qui garantissent ces services de régulation. Nous sommes donc d'emblée dans le cadre d'une analyse multicritères.

31 Les services de prélèvement permettent de produire des biens ou des services appropriables liés à la biodiversité : alimentation, eau douce, bois et fibres. La valeur de 
ces biens ou services peut être calculée d'un point de vue économique à un prix de marché ou à un coût de revient (cas de l'autoconsommation par exemple pour un jardin partagé sur une trame verte urbaine), en comparant la situation avec et sans la trame verte. Les services de prélèvement sont aussi limités par des seuils, la trame verte devant permettre un renouvellement des ressources dans un milieu donné.

Les services culturels permettent d'utiliser la trame verte à des fins récréatives, éducatives, esthétiques ou spirituelles. Les services éducatifs ou récréatifs peuvent être évalués monétairement par les coûts nécessaires à leur production, par les dépenses engagées pour les utiliser, par les marchés de substitution : coûts des déplacements pour accéder aux espaces récréatifs ou éducatifs par exemple. Cependant les services esthétiques ou spirituels ne peuvent pas être évalués monétairement car ils constituent une fin en soi pour les personnes. Ces services esthétiques et spirituels sont notamment liés à une biodiversité " remarquable " : habitats rares, paysages classés, éléments d'un héritage culturel. On peut dès lors suivre les propositions du Conseil d'Analyse Stratégique (CAS: 131) qui estime que " la biodiversité «remarquable» doit être considérée en prenant en compte les multiples critères, écologiques, éthiques, culturels, esthétiques ayant présidé à son identification et que de ce fait, l'analyse économique ne devra jouer dans ce cas qu'un rôle subsidiaire et indicatif (à l'image du cas des monuments historiques) ».

Dès lors, en prenant en compte les trois types de services écosystémiques d'une trame verte urbaine, on s'aperçoit qu'il faut combiner une évaluation monétaire et un ensemble de critères écologiques, éthiques et culturels. Nous sommes dès lors nécessairement dans le cadre d'une évaluation multicritères pluraliste, avec des points de vue différents, notamment des écologues scientifiques, des paysagistes et des utilisateurs de la trame verte, avec des indicateurs monétaires et non monétaires.

\subsection{La diversité des services liés à l'aménagement de la trame verte urbaine}

La trame verte urbaine correspond à de la biodiversité aménagée. En plus des services écosystémiques liés à la biodiversité, il faut prendre en compte dans l'évaluation socioéconomique d'une trame verte urbaine, les services liés à l'aménagement lui-même. Ces services dépendent des acteurs concernés qui vont utiliser ou développer ces services. Ces services peuvent être des services de transport, quand il s'agit de modes « actifs » comme nous l'avons mentionné dans la première partie de cet article, concernant des trajets pratiques ou des lieux agréables. Il s'agit là, d'un point de vue sociologique, de l'exploitation de la trame verte comme ressource naturelle aménagée dans le cadre des valeurs de la "cité industrielle qui poursuit sa mutation " (Lebot. et Philip, 2012). En suivant ce point de vue sociologique, dans le cadre des valeurs du "monde de l'opinion", où le renom d'un espace est lié aux médias, la trame verte peut valoriser des espèces rares, que l'on vient observer ou étudier; selon les valeurs du «monde domestique », la trame verte peut aussi valoriser un patrimoine local, en termes de faune, de flore, de recettes culinaires, de savoir-faire artisanaux. Toutes ces valorisations peuvent se traduire, d'un point de vue socio-économique, par des services touristiques, pour les visiteurs du territoire ayant aménagé une trame verte, si des acteurs du tourisme et des médias décident de fournir ces services et de les faire connaître. 
35 En plus des fonctions de base des écosystèmes concernés, caractérisées par des indicateurs écologiques, d'éléments sur les trois types de services écosystémiques, l'évaluation de la trame verte urbaine doit donc prendre en compte les services réels ou potentiels liés à l'aménagement lui-même, comme les services de transport et les services touristiques.

\subsection{La diversité des acteurs impliqués et les usages temporels de la trame verte urbaine}

La socio-économie des transports distingue les acteurs du transport, les administrations publiques et les tiers, en se centrant sur les acteurs du transport, à partir de modèles de prévision des trafics. L'utilisation de la trame verte urbaine peut difficilement être modélisée pour ce qui concerne ses utilisateurs, vu la diversité des usages possibles de la biodiversité. Il faut donc empiriquement répertorier les acteurs concernés par un projet de trame verte urbaine. La trame verte urbaine va alors se présenter comme une ressource naturelle aménagée utilisée par différents acteurs.

37 Le Conseil d'Analyse Stratégique (CAS, 2009 : 174) distingue différents temps d'usages des services écosystémiques, selon différents acteurs. Nous le suivrons sur ce point dans le cas des trames vertes urbaines. En effet, si l'objectif d'une trame verte urbaine est à la fois socio-économique (produire des flux de services écosystémiques aujourd'hui) et patrimonial (conserver un stock de patrimoine naturel pour les générations futures), il faut distinguer les services des écosystèmes de la trame verte urbaine pour les générations actuelles et ceux pour les générations futures. Le Conseil d'Analyse Stratégique distingue aussi les usages directs et les usages indirects.

Dans les usages pour les générations actuelles, on pourra différencier :

39 - les usages directs actuels de la trame verte urbaine (prélèvements de biomasse, loisirs, éducation, services culturels, services liés à l'aménagement) ;

40 - les usages indirects actuels de la trame verte urbaine, au travers d'effets utiles aux sociétés humaines actuelles concernant l'environnement naturel (fixation du carbone, rafraîchissement de l'air, diminution de la pollution locale, du bruit, épuration des eaux) ou l'environnement socio-économique (valorisation des bâtiments le long de la trame verte, pollinisation des jardins et des champs) ;

41 - la possibilité d'usages futurs pour les différents acteurs actuels de la trame verte urbaine suivant différentes options, soit parce que des incertitudes d'information seront levées (investissements envisagés pour des modes actifs comme la bicyclette par exemple), soit parce que ces usages futurs constituent une assurance contre des risques (prévention des inondations pour des riverains, possibilité de cultiver de nouvelles espèces pour des tiers ou des usagers en cas de difficultés économiques).

La possibilité d'usages futurs, selon différentes options, pour différents acteurs identifiés, ouvre celle d'usages futurs indéterminés par les générations à venir, avec éventuellement non-usage par les générations actuelles, afin de préserver les possibilités des générations futures. La valeur socio-économique totale des services écosystémiques et d'aménagement d'une trame verte urbaine comprendra donc dans le temps quatre composantes: pour les générations actuelles, une valeur d'usage directe, une valeur d'usage indirecte, une valeur d'option; et, pour les générations à venir, une valeur 
d'usages futurs (ou de transmission). Cette valorisation comportera des indicateurs monétaires et non monétaires.

\subsection{Les échelles d'aménagement (région urbaine, agglomération, quartier) et la possibilité d'une évaluation démocratique}

Les infrastructures de transport ont des effets essentiellement selon leur aire d'attraction (par exemple un effet d'attraction de la population sur un corridor d'environ 500 mètres, de part et d'autre, le long d'une ligne de tramway) et à l'intérieur des réseaux de transport (effet-réseau d'une nouvelle infrastructure complétant le réseau existant par de nouvelles connexions). Les trames vertes urbaines ont des effets à des échelles très diverses, de façon plus diffuse, car la faune et la flore peuvent migrer sur de vastes territoires ou se concentrer sur de très petits écosystèmes. Comme les projets de trames vertes urbaines doivent être surtout utiles aux sociétés humaines, tout en étudiant différentes échelles pour la faune et la flore, les échelles pertinentes d'évaluation socioéconomique des trames vertes nous semblent être celles de l'aménagement urbain: le quartier, l'agglomération, la région urbaine. Ces échelles peuvent s'imbriquer dans des échelles plus vastes : l'échelle de la planète pour les évaluations concernant le $\mathrm{CO}_{2}$ par exemple.

Ces échelles d'aménagement sont notamment celles que privilégie le mouvement du « New Urbanism » aux États-Unis, qui avait proposé la notion de « corridor » comme outil d'aménagement dans sa Charte. Les corridors du « New Urbanism » peuvent être soit des corridors pour les transports collectifs (« transit corridors »), soit des corridors d'espaces naturels ou de jardins, reliant les différents quartiers des villes, les distinguant les uns des autres, fournissant " un lien universel avec la nature » (Congress for the New Urbanism, 1999: 113-119). Nous retrouvons là des éléments de justification des trames vertes urbaines, selon un point de vue éthique et culturel. Ces échelles sont, en France, le lieu d'institutions de démocratie locale : conseils de quartier, communautés d'agglomération, conseils régionaux. L'évaluation des trames vertes urbaines, avec ses différents indicateurs, usages et acteurs concernés, peut donc, par ses enjeux, contribuer à animer la démocratie locale.

\section{Conclusion}

L'application des méthodes d'évaluation des infrastructures de transport aux trames vertes urbaines nous a conduit, dans un premier temps, à prendre en compte une évaluation monétaire ou monétarisée par différentes méthodes (marchés de substitution avec notamment les prix hédoniques, évaluations contingentes, coûts indirects des dommages ou d'évitement des dommages) pour quantifier avec un équivalent monétaire commun les services de la trame verte urbaine. Cette valeur monétaire ou monétarisée est comparée aux coûts de l'aménagement de la trame verte pour la collectivité territoriale concernée (coûts d'investissement et coûts de fonctionnement) pour dégager un bénéfice social actualisé, dans le cadre d'une analyse coûts-avantages. Cette évaluation, basée sur des éléments techniques et scientifiques précis, correspond à une évaluation «managériale» (Goxe, 2010: 71-81). Elle permet aux acteurs concernés par l'aménagement, et en premier lieu à la collectivité territoriale en charge du projet, de 
gérer le projet et de le justifier économiquement, quitte à l'imposer ensuite d'autorité aux autres acteurs.

Nous avons vu que ce type d'évaluation coûts-avantages atteint ses limites, dans le cas des infrastructures de transport, car elle ne prend pas en compte le jeu potentiel des acteurs et la mise en système des nuisances liées au transport automobile. Elle fournit cependant des éléments méthodologiques intéressants pour évaluer les trames vertes urbaines: méthodes d'évaluation monétaire pour le bruit, la pollution locale de l'air ou les émissions de $\mathrm{CO}_{2}$; comparaison de deux situations avec ou sans aménagement ; rôle de la trame verte dans le cadre des déplacements de personnes et des biens.

Cependant, dans le cas des trames vertes, ce type d'évaluation atteint encore plus fortement ses limites que dans le cas des infrastructures de transport: les trames vertes nécessitent des indicateurs écologiques, mettent en jeu une grande diversité d'acteurs en interaction avec des finalités contradictoires, mobilisent des valeurs culturelles, paysagères et esthétiques, dans un cadre spatialisé. Elles nécessitent d'emblée des évaluations monétaires et non monétaires, donc une évaluation multicritères spatialisée.

Dans le cadre d'objectifs généraux de développement durable, comme pour les trames vertes et d'autres projets où l'environnement naturel est concerné, il existe, d'après Antoine Goxe, en plus de l'évaluation "managériale », deux autres types d'évaluation: une évaluation " pluraliste » et une évaluation « démocratique ».

L'évaluation pluraliste résulte du croisement de points de vue différents, avec des acteurs aux intérêts parfois divergents. Les expertises issues des savoirs et celles issues du vécu des acteurs concernés sont alors mutuellement reconnues pour aboutir à un point de vue commun. Ce point de vue, d'abord qualitatif, peut être éventuellement quantifié par un indicateur. Ce type d'évaluation pluraliste s'impose pour les trames vertes urbaines, si l'on prend en compte leur objectif de préservation et d'augmentation de la biodiversité en lien avec le maintien des fonctions de base des écosystèmes, étudiées par les écologues, et le caractère non monétaire de certains usages des trames vertes urbaines, selon des valeurs esthétiques ou spirituelles.

En étudiant les services et les fonctions des trames vertes urbaines, cinq types d'éléments, avec des indicateurs monétaires ou non monétaires nous semblent devoir figurer dans une grille d'évaluation des trames vertes urbaines : les fonctions de base des écosystèmes concernés, avec des indicateurs écologiques, les services écosystémiques de régulation, d'approvisionnement et culturels, les services liés à l'aménagement. L'aménagement d'une trame verte dépendra de choix entre différentes valeurs, avec de fortes incidences spatiales, donc d'une évaluation pluraliste multicritères spatialisée.

51 Selon Antoine Goxe (2010), l'évaluation démocratique suppose une négociation entre décideurs et opérateurs des politiques publiques d'une part (par exemple la collectivité territoriale et les différentes entreprises ou administrations concernées pour les trames vertes) et, d'autre part, les citoyens impliqués, par exemple par le biais d'associations. Une concertation entre ces acteurs, sur la base de règles communes, devrait pouvoir dégager des éléments communs d'évaluation.

Les acteurs de cette évaluation, qui sont nettement cités dans le cas des infrastructures de transport (usagers, tiers, administrations publiques) sont moins clairement identifiés dans le cas des trames vertes urbaines. En effet, l'institution d'un collectif d'acteurs concernés et la fabrication de règles communes entre ces acteurs sont, dans le cas de ressources naturelles communes comme les trames vertes, «un processus difficile, 
chronophage et propice aux conflits. C'est un processus qui requiert une information fiable en termes de variables de temps et de lieu, ainsi qu'un vaste répertoire de règles culturellement acceptables » (Ostrom, $2010: 27$ ).

L'évaluation des trames vertes urbaines est donc liée à la gouvernance des acteurs concernés par ces trames vertes urbaines pour définir des règles communes efficaces par rapport aux objectifs affichés concernant la biodiversité. Cette gouvernance pourra élargir les méthodes d'évaluation pour conduire, au-delà des évaluations "pluralistes " nécessaires, à des évaluations "démocratiques ", en fonction de l'importance des enjeux de la trame verte et de l'implication des acteurs concernés. Ce thème de recherche sur la gouvernance des acteurs des trames vertes urbaines pourra utiliser notamment les apports de la politique « néo-institutionnelle » d'Elinor Ostrom, où l'auto-organisation et l'auto-gouvernance sont privilégiées dans des situations de ressources naturelles communes de petite échelle.

Une réflexion devient alors nécessaire sur les échelles d'évaluation. Nous avons proposé de partir des échelles des aménageurs, en insistant sur le caractère urbain des trames vertes étudiées. Nous avons pensé que les trames vertes urbaines sont un des éléments de la ville durable en gestation (Masboungi, 2008; Charmes, Souami, 2009). Elles y ont, à notre avis, un rôle important. Elles peuvent servir à tisser un lien entre les quartiers des villes, comme le propose le mouvement du «New Urbanism». Mais ces liaisons interquartiers ne suffisent pas à définir toutes les potentialités des trames vertes urbaines dans le nouvel aménagement des villes. Car les trames vertes urbaines peuvent permettre de trouver un nouveau rapport, autour de l'enjeu de la biodiversité, en particulier de la biodiversité locale vue comme un patrimoine sur la longue durée, entre les trois espaces fondamentaux d'une région urbaine: les espaces urbains, les espaces ruraux et les espaces naturels.

Les trames vertes urbaines posent alors des questions d'imbrication des échelles ou des niveaux d'évaluation, avec des règles différenciées selon les échelles. Cette question d'imbrication des échelles et des règles n'est pas insurmontable : «lors d'une analyse à quelque niveau que ce soit, et pour les besoins de celle-ci, l'analyste doit fixer les variables d'un niveau supérieur. (...) La stratégie clé des individus auto-organisés et autogouvernés, pour faire face aux problèmes auxquels ils sont confrontés sur le terrain, consiste à aller et venir entre les différents niveaux » (Ostrom, $2010: 72)$. L'évaluation et la gouvernance des trames vertes urbaines seraient donc une incitation à mettre en place de nouvelles formes démocratiques participatives à différentes échelles de la région urbaine.

\section{BIBLIOGRAPHIE}

Bérion, Pascal et al., 2007, « L'évaluation socio-économique des infrastructures de transport: enrichir les approches du développement territorial ", Revue d'Économie Régionale et Urbaine, 4/2007 (novembre), p. 651-676. 
Centre d'Analyse Stratégique, 2009, Approche économique de la biodiversité et des services liés aux écosystèmes, Mission présidée par Bernard Chevasses-au-Louis, Paris, La Documentation française, $224 \mathrm{p}$.

Charmes E., Souami T., 2009, Villes rêvées, villes durables ? Paris, Gallimard, 32 p.

Commissariat Général du Développement Durable, 2010, Conservation et utilisation durable de la biodiversité et des services écosystémiques, analyse des outils économiques, Paris, $240 \mathrm{p}$.

Commissariat Général du Développement Durable, mai 2010, Projet de caractérisation des fonctions écologiques des milieux en France, Paris, 70 p.

Commissariat Général du Développement Durable, juillet 2011, Schéma National des Infrastructures de Transport (avant-projet consolidé), Rapport environnemental, Paris, $302 \mathrm{p}$.

Commissariat Général du Plan, 2001, Transports : choix des investissements et coûts des nuisances, Paris, La Documentation française, $328 \mathrm{p}$.

Congress for the New Urbanism, 1999, Charter of the New Urbanism, New York, Mc Graw-Hill, p. 113-119.

Conseil Général des Ponts et Chaussées, 2005, Analyse comparative des méthodes d'évaluation des grandes infrastructures de transport, Groupe de travail présidé par Claude Gressier, Paris, La Documentation française, $53 \mathrm{p}$.

Conseil National des Transports, 1999, Les transports et l'environnement, vers un nouvel équilibre, Rapport du groupe de travail présidé par Alain Bonnafous, Paris, La Documentation française, p. 19-47.

Goxe A., 2010, «L'évaluation des politiques territoriales au regard du développement durable » in Zuindeau B. (dir.), Développement durable et territoire, Villeneuve d'Ascq, Presses Universitaires du Septentrion, p. 71-81.

Héran F., 2011, « Pour une approche systémique des nuisances liées aux transports en milieu urbain », in Les Cahiers Scientifiques du Transport, n 59, p. 83-112.

Le Bot J.-M., Philip F., « Les trames vertes urbaines, un nouveau support pour une cité verte? », Développement durable et territoires [En ligne], vol. 3, $\mathrm{n}^{\circ} 2$ | Juillet 2012, mis en ligne le 06 juillet 2012, consulté le 23 juillet 2012. URL : http://developpementdurable.revues.org/9318

Masboungi A., 2008, Breda, faire la ville durable, Paris, Le Moniteur, 144 p.

Millenium Ecosystem Assessment, 2005, Ecosystems and Human Well-being, Biodiversity Synthesis, Washington DC, World Resources Institute, 86 p.

Ministère de l'Équipement, des Transports, du Logement, 2005, Instruction cadre relative aux méthodes d'évaluation économique des grands projets d'infrastructures de transport, 25 mars 2004, mise à jour le 27 mai 2005.

Ostrom E., 2010, Gouvernance des biens communs, pour une approche nouvelle des ressources naturelles, Bruxelles, De Boeck, 301 p.

Quinet E., 1998, Principes d'économie des transports, Paris, Economica, p. 149-152.

Schiller Preston L., Bruun Eric C., Kenworthy Jeffrey R., 2010, An introduction to sustainable transportation : policy, planning and implementation, Londres, Earthscan, p. 153-188.

Stambouli J., 2000, « Développement soutenable et valorisation des externalités environnementales des transports », Les Cahiers Scientifiques du Transport $n^{\circ}$ 38, p. 63-94.

Site web de l'ANR Trame Verte urbaine : www.trameverteurbaine.com. 


\section{RÉSUMÉS}

La terminologie employée pour la définition des trames vertes urbaines (projet, infrastructures aménagées, flux, corridors, amélioration du cadre de vie, collectivités territoriales) rappelle celle des infrastructures de transport, éléments fixes d'un réseau permettant la circulation des personnes et des biens dans des territoires. Cet article examine l'apport des différentes méthodes socio-économiques d'évaluation des infrastructures de transport pour la prise en compte, par les aménageurs, des trames vertes urbaines. Il pointe les limites de ces méthodes pour les trames vertes urbaines, du fait de la pluralité des services écosystémiques liés à la biodiversité, de la diversité des acteurs concernés et des échelles d'aménagement. Il préconise une méthode d'évaluation multicritères pluraliste spatialisée, avec des indicateurs monétaires et non monétaires et envisage les conditions d'une évaluation démocratique.

The terminology used for the definition of urban greenways (project, developed infrastructure, flows, corridors, improvement of living conditions, local authorities) is reminiscent of the transport infrastructures, fixed elements of a network for the movement of people and goods inside territories. This article examines the contribution of socio-economic evaluation methods of transport infrastructures to evaluate, by developers, the urban greenways. It highlights the limits of these methods for the urban greenways, because of the plurality of ecosystem services related to biodiversity, the diversity of stakeholders and scales of development. It advocates a spatial pluralistic Multi-criteria evaluation method, with monetary and non-monetary items, and takes into consideration the conditions for a democratic evaluation.

\section{INDEX}

Mots-clés : trame verte urbaine, socio-économie, infrastructures de transport, évaluation multicritères, services écosystémiques, biodiversité

Keywords : urban greenway, Socio-economics, transport infrastructures, Multi-criteria evaluation, ecosystem services, biodiversity

\section{AUTEUR}

\section{JACQUES STAMBOULI}

Jacques Stambouli est docteur en sciences économiques, maître de conférences en sciences de gestion à l'Université d'Artois (Laboratoire DYRT, EA 2468) et chercheur associé au CRIA (UMR 8504, Géographies-cités) de l'Université Paris 1. Publication récente : « Les services de transport du développement urbain durable : innovations autour des métriques de la marche à pied », in Revue de l'Économie Méridionale, 1-2/2009 vol. 57, n²25-226 p. 129-156, Université de Montpellier. Ses domaines de recherche sont la socio-économie de la ville durable, la socioéconomie des transports et l'histoire socio-économique des espaces urbains sur la longue durée. Contact : stambouli.j@orange.fr. L'auteur remercie les responsables du programme de recherche ANR-Trame Verte Urbaine, Nathalie Blanc et Philippe Clergeau, pour l'avoir accueilli comme observateur de certains de leurs travaux. 